\title{
Navigating the Jargon of Molecular Testing in Non-Hodgkin Lymphoma
}

\author{
Presented by Eric D. Hsi, MD
}

\begin{abstract}
The many different subtypes of non-Hodgkin lymphoma can be distinguished by histopathology, immunophenotype, and underlying genetic abnormalities. Molecular testing is routinely used by pathologists for diagnosis and prognosis, and to aid in guiding therapy selection (targeted or nontargeted). The different tools and techniques used for molecular testing are based on abnormality type and tissue available.

J Natl Compr Canc Netw 2020;18(12.5):1745-1747 doi: $10.6004 /$ jnccn.2020.5032
\end{abstract}

We all know that non-Hodgkin lymphoma is not just one lymphoma, said Eric D. Hsi, MD, Chair of the Department of Laboratory Medicine, Professor of Pathology at Lerner College of Medicine, and member of Case Comprehensive Cancer Center/University Hospitals Seidman Cancer Center and Cleveland Clinic Taussig Cancer Institute, at the NCCN 2020 Virtual Congress: Hematologic Malignancies. The WHO classification includes 42 main entries for B-cell malignancies and 27 for mature T-cell and NK-cell neoplasms alone. ${ }^{1}$

"I spend a lot of my time staring down the barrel of a microscope, wondering what the diagnosis is," said Dr. Hsi. "We've come a long way from several decades ago when all we had was routine [hematoxylin \& eosin] sections, and we're now able to apply different technologies to examine cell proteins on cell surfaces or within cells by flow cytometry, where we label cells with fluorescent antibodies. We can do this now with 10 to 12 antibodies at the same time, analyzing thousands of cells within a few seconds, and coming up with a characteristic phenotype of a lymphoma cell."

According to Dr. Hsi, remembering the central dogma of molecular biology can help cut through the confusion in classifying lymphoma subtypes: techniques to detect DNA, RNA, and protein should be applied to help characterize lymphoid malignancies and to answer specific diagnostic, prognostic, and therapeutic questions. DNA makes up the building blocks of genetic information and can undergo replication. DNA is then transcribed to RNA, which is then translated into protein. "The ancillary studies that we perform will look at one or multiple of these types of molecules: DNA, RNA and protein. We use these to characterize lymphoid malignancies, make a diagnosis, prognosticate, and sometimes make therapeutic decisions," he said. "It can be confusing, but if we remember the central dogma of molecular biology, we can take stock of what we're looking at and make sense of it."

\section{What Types of Molecular Abnormalities Do We Look For?}

In DNA, gene copy number abnormalities (gains or losses) and structural variants (translocations/rearrangements) can be analyzed, as well as mutations (small DNA sequence changes). These often present as single nucleotide variants or insertions or deletions of small numbers of base pairs within a gene. These mutations can cause gain or loss of function of the gene, and can regulate transcription of the gene or stability of the RNA transcript. Expression of genes (RNA) or gene products (proteins) can also be evaluated, simply for presence, or quantified by the amount of RNA or protein expressed.

Copy number gains and losses can be analyzed using a number of techniques, including routine karyotyping, fluorescence in situ hybridization (FISH), DNA microarrays, or next-generation sequencing (NGS). Structural variants, characterized by the movement of genes or chromosomes within the genome, can be found through routine karyotyping, FISH, PCR-based assays (either at the DNA or RNA level via reverse-transcriptase PCR [rtPCR]), and NGS-based methods.

Methods for identifying mutations can be used in both fresh and formalin-fixed paraffin-embedded (FFPE) tissues. "Sanger sequencing is an older technology and is a good method when you don't really know exactly what the mutation is but you want to sequence one small area of a gene," he noted. "You can get the whole sequence this way and determine the abnormality." Other techniques include allele-specific PCR-useful 


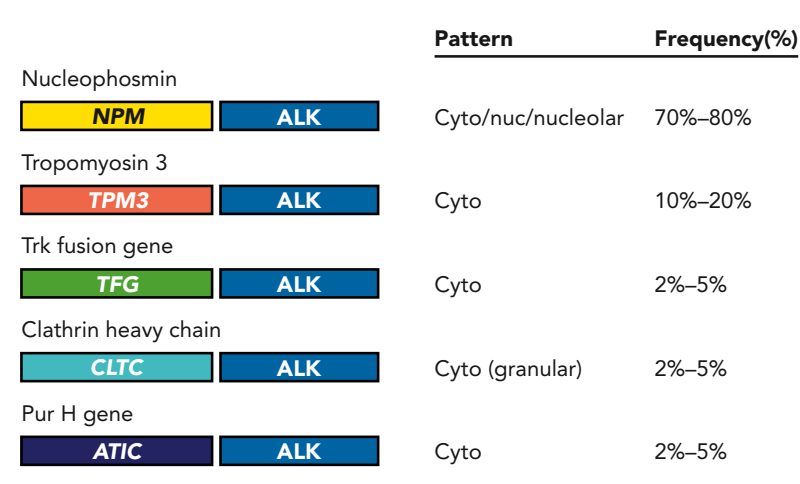

Figure 1. Variant ALK translocations.

Abbreviations: cyto, cytoplasmic; nuc, nuclear.

when looking for a highly recurrent, specific mutation-and PCR and fragment-length analysis, used to find insertions and deletions. Although these methods typically examine one gene or small fragment of a gene at a time, NGS-based methods are very high throughput, and can analyze hundreds or thousands of genes simultaneously.

Gene expression relates to RNA detection, which can be performed using rtPCR with fresh or FFPE tissue (quantitative, looking at a single or few targets), gene expression array with fresh or frozen tissue (somewhat quantitative, looking at hundreds/thousands of genes), multiplex molecular digital barcoding technology with fresh or FFPE tissue (looking at scores/hundreds of genes), or RNA-sequencing by NGS with fresh or FFPE tissue (can look at thousands of genes at once).

Protein expression can be measured with flow cytometry or immunohistochemistry. "With flow cytometry, we can use fresh or cryopreserved cells, stain them simultaneously with 10 to 12 different markers, and get information about surface proteins," he said. "With immunohistochemistry, we can usually use 1 or 2 markers at a time, but we get in situ (spatial) information as to where these cells are in relation to one another."

\section{Treating Lymphoma Subtypes}

Dr. Hsi expanded on the current treatment modalities available for selected illustrative lymphoma subtypes.

Anaplastic large cell lymphoma (ALCL) is an aggressive peripheral T-cell lymphoma derived from cytotoxic $\mathrm{T}$ cells that strongly and uniformly expresses CD30 (which is itself a target for therapy). Anaplastic lymphoma kinase (ALK)-positive and ALK-negative tumors in ALCL must be distinguished from one another because they have vastly different prognoses, with ALKpositive cases being favorable.

ALK (a tyrosine kinase) is also targetable with ALK inhibitors, so it is important to know whether ALK is translocated in ALK-positive ALCL, resulting in overexpression

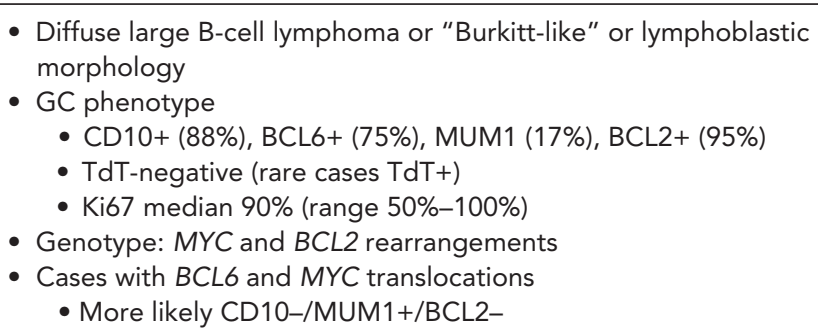

Figure 2. Pathologic features of double-hit lymphoma (high grade B-cell lymphoma with MYC and BCL2 and/or BCL6 rearrangement).

of the ALK protein. This molecular abnormality can be detected by immunohistochemistry; normal cells do not express ALK, but cells with this translocation overexpress it.

Brentuximab vedotin, an antibody-drug conjugate against CD30, is the preferred regimen for ALCL and other CD30-positive T-cell lymphoma histologies, according to the NCCN Guidelines for T-Cell Lymphomas, ${ }^{2}$ and should be used in combination with other chemotherapeutic agents. ${ }^{2}$

Fusion of ALK to NPM most commonly results in $A L K$ expression in ALCL. "But we've learned over time that there are other partner genes for $A L K$ fusions, and they result in a different immunostaining pattern," he said. "We can actually get information about particular gene partners just from the immunohistochemical pattern" (Figure 1).

Double-hit lymphoma (DHL) is an aggressive type of B-cell non-Hodgkin lymphoma characterized by rearrangements in 2 particular genes (usually MYC and $B C L 2$; Figure 2). "These have a germinal center B-cell phenotype, they express CD10, and are TdT-negative with a high proliferative index," Dr. Hsi explained. "We also now accept some cases that have BCL6/MYC translocations."

According to Dr. Hsi, it is important to recognize DHL, because this malignancy presents at a more advanced stage in middle-aged and older adults, resulting in a poor prognosis. Retrospective studies of DHL show a very short median survival of usually $<2$ years, but research suggests that intensified induction chemotherapy leads to better outcomes than standard rituximab $\mathrm{CHOP}$ (cyclophosphamide/doxorubicin/vincristine/prednisone) chemotherapy, he noted. ${ }^{3,4}$

New research in follicular lymphoma has recently led to the accelerated approval of an EZH2 inhibitor. This FDA approval was based on overall response rate (ORR) and duration of response in a trial of adult patients with relapsed/refractory follicular lymphoma whose tumors harbor an EZH2 mutation (present in approximately $20 \%-25 \%$ of follicular lymphomas), had received 
$\geq 2$ prior systemic therapies, or those with relapsed/ refractory follicular lymphoma who had no satisfactory alternative treatment options. ORRs were $69 \%$ in patients with EZH2 mutations compared with $35 \%$ in those who were wild-type, with a median duration of response of approximately 11 and 13 months, respectively. ${ }^{5}$

The cobas EZH2 Mutation Test (Roche Diagnostics) companion diagnostic is a real-time, allele-specific PCR test available for qualitative detection of recurrent hotspot mutations. "This is now suitable for DNA extracted from FFPE tissue," said Dr. Hsi. "It's another example of where detecting a molecular abnormality would help select therapy for patients."

Disclosures: Dr. Hsi has disclosed that he has received grant/research support from AbbVie, Inc. and Eli Lilly and Company, and honoraria from Miltenyi Biotech and Seattle Genetics, Inc.

Correspondence: Eric D. Hsi, MD, Department of Laboratory Medicine, Cleveland Clinic, 9500 Euclid Avenue, L-30, Cleveland, OH 44195. Email: hsie@ccf.org

\section{References}

1. Swerdlow SH, Campo E, Harris NL, et al. WHO Classification of Tumours of Haematopoietic and Lymphoid Tissues, 4th edition. Lyon, France: IARC Press; 2017.

2. Horwitz SM, Ansell S, Ai WZ, et al. NCCN Clinical Practice Guidelines in Oncology: Primary Cutaneous Lymphomas. Version 1.2021. Accessed October 20, 2020. Available at: NCCN.org

3. Aukema SM, Reiner S, Schuuring E, et al. Double-hit B-cell lymphomas. Blood 2011;117:2319-2331.
4. Petrich AM, Gandhi M, Jovanovic B, et al. Impact of induction regimen and stem cell transplantation on outcomes in double-hit lymphoma: a multicenter retrospective analysis. Blood 2014;124: 2354-2361.

5. Morschhauser F, Tilly $H$, Chaidos $A$, et al. Phase 2 multicenter study of tazemetostat, an EZH2 inhibitor, in patients with relapsed or refractory follicular lymphoma [abstract]. Blood 2019;134(Suppl 1): Abstract 123 